\title{
THE CURRENT STATE AND THE DEVELOPMENT PROSPECTS OF THE UKRAINIAN MARKET OF ANTIHISTAMINES
}

Nowadays allergy has become a global problem for all humanity. According to the WHO about 30-40 \% of the world's population suffers from allergic diseases. Environment pollution, as well as everyday use of a large number of synthetic substances, contribute to the rapid increase in incidence of allergic reactions. The therapy of these diseases is based mainly on the antihistamine drugs (AHD), which makes it reasonable to study the Ukrainian market of AHD and to identify its promising directions of development.

Aim. To study the assortment of antihistamine drugs registered in Ukraine.

Materials and methods. The analysis was based on the official sources of information and materials of the previous marketing research using the methods of systematic, graphical and comparative analysis with the subsequent obtaining of explanations and conclusions.

Results. During the analysis of the domestic pharmaceutical market regarding the assortment of antihistamines it has been found that 128 drugs are used in Ukraine for the treatment of allergic diseases. The study of distribution by dosage forms has revealed the prevalence of solid dosage forms - tablets and dragee - their percentage is $71 \%$. The analysis of AHD by the manufacturing countries has shown the import dependence of the Ukrainian market: the foreign manufacturers represent $61 \%$ of the drugs. Moreover, in recent years there is a downward trend of the share of domestic manufacturers $(50 \%-$ in $2014 ; 39 \%$ - in 2019). India is the largest importer of antihistamines at the market (17.2\%); the total market share of the European Union countries is $39 \%$. The analysis of the current state of the pharmaceutical market has determined a change in the distribution of AHD by INN in favor of the new-generation drugs. The study has revealed the absence of anti-allergic herbal medicines in Ukraine; it substantiates the development of new drugs for the phytotherapy of allergic diseases.

Conclusions. The absence of plant-based anti-allergic drugs at pharmaceutical market of Ukraine has been found. Taking into account an increasing popularity of herbal medicines and growing demand for drugs with a good safety profile we have concluded that the development of a new plant-based drug for the allergy treatment is a topical direction for further studies.

Key words: allergic diseases; pharmaceutical market; antihistamines.

\section{О. В. АндРюШАєв, О. А. РуБАН, Ю. С. МАсЛІй, Л. В. ЯковЛєВА \\ Національний фармацевтичний університет, Украӥна \\ СУЧАСНИЙ СТАН ТА ПЕРСПЕКТИВИ РОЗВИТКУ РИНКУ АНТИГІСТАМІННИХ ПРЕПАРАТІВ в УКРАїні}

У наш час алергія стала глобальною проблемою для всього людства. За даними В003, близько 30-40 \% населення земної кулі страждають від алергічних захворювань. Забруднення зовнішнього середовища разом із частим використанням у побуті великої кількості синтетичних речовин сприяють стрімкому розповсюдженню алергічних реакцій. Терапія цих захворювань грунтується більшою мірою на застосуванні антигістамінних препаратів (АГП), що обумовлює доцільність дослідження ринку АГП в Україні та виявлення пріоритетних напрямів його розвитку.

Мета: дослідження асортименту зареєстрованих в Україні антигістамінних лікарських засобів.

Матеріали та методи. Аналіз проводився на основі офіційних джерел інформації та матеріалів попередніх маркетингових досліджень із використанням методів системного, графічного і порівняльного аналізу та наступним одержанням пояснень і висновків.

Результати дослідження. У ході аналізу вітчизняного фармацевтичного ринку щодо асортименту антигістамінних лікарських засобів було встановлено, що в Україні зареєстровано 128 препаратів, які застосовуються для лікування алергічних захворювань. Дослідження розподілу за лікарськими формами виявило перевагу твердих лікарських форм - таблетки і драже становлять $71 \%$. Аналіз АГП за країнами-виробниками показав імпортозалежність ринку - 61 \% препаратів представлено імпортними виробниками. При цьому останнім часом спостерігається тенденція до зменшення частки вітчизняного виробника (50 \% - у 2014 р.; $39 \%$ - у 2019 р.). Найбільшим імпортером антигістамінних лікарських засобів на ринок $є$ Індія $(17,2 \%)$; сумарний внесок країн Європейського Союзу становить 39 \%. Аналіз сучасного стану фармацевтичного ринку виявив зміну розподілу АГП за МНН на користь лікарських засобів нового покоління. У ході дослідження виявлено відсутність в Україні протиалергічних лікарських засобів рослинного походження, що дає підстави для розробки нових препаратів для фітотерапії алергічних захворювань. 
Висновки. Установлено відсутність протиалергічних рослинних препаратів на фармацевтичному ринку України. Враховуючи зростаючу популярність рослинних лікарських засобів та високий рівень попиту на лікарські засоби з хорошим профілем безпеки, ми дійшли висновку, що розробка нового протиалергічного препарату на рослинній основі є актуальним напрямком подальших досліджень.

Ключові слова: алергічні захворювання; фармацевтичний ринок; антигістамінні лікарські засоби.

\section{А. В. АНДРЮШАЕВ, Е. А. РУБАН, Ю. С. МАСЛИЙ, Л. В. ЯкОВЛЕВА \\ Национальный фармацевтический университет, Украина \\ СОВРЕМЕННОЕ СОСТОЯНИЕ И ПЕРСПЕКТИВЫ РАЗВИТИЯ РЫНКА АНТИГИСТАМИННЫХ ПРЕПАРАТОВ В УКРАИНЕ}

В наше время аллергия стала глобальной проблемой для всего человечества. По данным В03, около 30-40 \% населения земного шара страдают от аллергических заболеваний. Загрязнения внешней среды вместе с частым использованием в быту большого количества синтетических веществ способствуют стремительному распространению аллергических реакций. Терапия этих заболеваний основывается в большей степени на применении антигистаминных препаратов (АГП), что обусловливает целесообразность исследования рынка АГП в Украине и выявления приоритетных направлений его развития.

Цель: исследование ассортимента зарегистрированных в Украине антигистаминных лекарственных средств.

Материалы и методы. Анализ проводился на основании официальных источников информации и материалов предварительных маркетинговых исследований с использованием методов системного, графического и сравнительного анализа и последующим получением объяснений и выводов.

Результаты исследования. В ходе анализа отечественного фармацевтического рынка по ассортименту антигистаминных лекарственных средств было установлено, что в Украине зарегистрировано 128 препаратов, применяемых для лечения аллергических заболеваний. Исследование распределения по лекарственным формам выявило преимущество твердых лекарственных форм - таблетки и драже насчитывают 71 \%. Анализ АГП по странам-производителям показал импортозависимость рынка - 61 \% препаратов представлен импортными производителями. При этом за последние годы наблюдается тенденция к уменьшению доли отечественного производителя (50 \% - в 2014 г.; 39 \% - в 2019 г.). Крупнейшим импортером антигистаминных лекарственных средств на рынок является Индия (17,2 \%); суммарный вклад стран Европейского Союза составляет 39 \%. Анализ современного состояния фармацевтического рынка установил изменение распределения АГП по МНH в пользу лекарственных средств нового поколения. В ходе исследования выявил отсутствие в Украине противоаллергических лекарственных средств растительного происхождения, что обосновывает разработку новых препаратов для фитотерапии аллергических заболеваний.

Выводы. Установлено отсутствие антиаллергических препаратов растительного происхождения на фармацевтическом рынке Украины. Принимая во внимание растущую популярность растительных лекарственных средств и високий спрос на лекарства с хорошим профилем безопасности, мы пришли к выводу, что разработка нового растительного лекарственного средства для лечения аллергии является актуальным направлением для дальнейших исследований.

Ключевые слова: аллергические заболевания; фармацевтический рынок; антигистаминные лекарственные препараты.

Statement of the problem. Human modern life is inseparably connected with the daily use of a large number of synthetic substances. Environment pollution by industrial waste, use of chemicals in farming and daily life, the frequent use of dyes and preservatives in the food and pharmaceutical industry negatively affect the health and lead to the different types of allergic reactions. The upward trend in the incidence of allergy is also caused by nutrition disorders, uncontrolled medication, various chronic diseases and long-term stress, which are an integral part of the human existence $[1,2]$.

To date, about $30-40 \%$ of the world's population suffers from the allergic diseases. In addition, statistics indicate a rapid increase in the incidence of allergic reactions over the last
50 years, especially in countries with the prowestern lifestyle [1]. There are no official data of these diseases in Ukraine, but according to literature sources, the number of patients is about $20-30 \%$, confirming the relatively high incidence of allergies in our country.

This problem is especially acute in pediatric practice. In recent years, there has been a steady increase in the incidence of allergic reactions in children and adolescents. The risk of allergy sensitization of the young immune system is much higher; therefore, it is sometimes very difficult to predict the risk of allergy in children. That is why doctors often recommend the prophylactic therapy, which has specific requirements for efficacy and safety [3-7]. 
In the modern therapeutic practice, the allergic reactions are mainly treated due to the effect on histamine receptors and the metabolism of histamine. There are two main approaches: 1) reducing free histamine; 2) blockade of histamine receptors. By the first approach the activity of the group of drugs called "stabilizers of mast cells membranes" (ketotifen, cromoglycic acid, nedocromil, etc.) is effective. They are widely used in medical practice, but their moderate and long-lasting effect allows using them for prophylactic purposes only. The group of antihistamine drugs (AHD) has faster and stronger anti-allergic activity. Thus, this group is the first line therapy in the treatment of most allergic diseases [8].

The modern AHD classification includes 3 generations. The first-generation drugs (diphenhydramine, clemastine, chloropheniramine, mebhydrolin, etc.) have been used in clinical practice for many years; it gives them an advantage in terms of the wide experience of their application. A major disadvantage is their ability to easily dissolve in lipids, pass through the blood - brain barrier (BBB), and bind to histamine, serotonin, dopamine, and m-acetylcholine receptors of the brain, affecting the central nervous system (CNS). $40-80 \%$ of patients subjectively notice various types of cognitive impairment: drowsiness, anxiety, nervousness, dizziness, vestibular disorders, hypotension, impaired learning ability, memory impairment, etc. In addition, the first AHD generation also has a short-term effect and provides development of tachyphylaxis.

The $2^{\text {nd }}$ generation of AHD (loratadine, dimethyndene, cetirizine) do not pass through the BBB and have a long and sustainable effect. But pharmacokinetic peculiarities cause the accumulation of their metabolites in the tissues of the heart and provide the cardiotoxic effect (except loratadine) [8-11].

The $3^{\text {rd }}$ AHD generation includes active metabolites and stereoisomers of drugs of the previous generation (desloratadine, levocetirizine, fexofenadine), which are characterized by the best safety profile among other AHD groups $[8,10,11]$.

Therefore, the wide range of applications and the peculiar pharmacological actions of each generation of this group of drugs allow us to state that AHD are indispensable for the treatment of allergic reactions of different complexity and etiology.

Analysis of recent research and publications. The studies of the domestic pharmaceutical market of AHD in different periods were carried out by L. V. Yakovleva, M. O. Syzenko, O. G. Berdnik, A. O. Gurtyakova etc. [12-14].

Identification of aspects of the problem unsolved previously. In 2019, at the Department of Industrial Technology of Drugs of the National University of Pharmacy the research on developing of a new anti-allergic medicine based on a complex plant extract began. The new drug is planned to be created in the form of tablets containing dry extracts of the following plants: Bidens tripartita, Calendula officinalis and Cretaegus sanguinea in the established ratio. Since the development of a new drug always requires the identification of its potential importance for the consumer, conducting the market research in order to identify the state and the potential directions of the AHD market development is an expedient and relevant task.

Objective statement of the article. The aim of our work was to analyze the assortment of antihistamines available at the pharmaceutical market of Ukraine.

Materials and methods. The analysis was based on the official sources of information, such as the State Register of Medicines of Ukraine, the directory "Compendium 2018 - medicines" and materials of the previous marketing research $[12,13]$. The methods of systematic, graphical and comparative analysis were used to obtain explanations and conclusions.

In addition, the AHD affordability for the population of Ukraine was determined. The number of dosage units for the calculation of the indicator was chosen based on the average duration of the treatment of chronic allergic diseases, i.e. 5 weeks $[12,15]$. The solvency index (Ca.s., \%) was calculated using the formula (1):

$$
\text { Ca.s. }=\text { P / Wa.w. } \cdot 100 \%
$$

where $\mathrm{P}$ - is the average cost of the treatment course as of November 2019; Wa.w. - is the average salary as of November 2019.

The average salary was determined according to official statistics [16].

The solvency index Ca.s. was interpreted due to distribution of trade names into 3 groups: 
highly affordable if the value of the index Ca.s. was less than $5 \%$; medium affordable if Ca. s. was in the range of 5-15\% and unaffordable where Ca.s. was above $15 \%$ [17].

Presentation of the main material of the research. As of November 2019, the R06A group "Antihistamine drugs" (according to the ATCclassification) accounted for 128 drugs, which are used to treat allergic diseases, at the pharmaceutical market in Ukraine (Table) $[18,19]$.

By the results presented in Table the study of the variability of dosage forms (DF), in which
AHD were prepared, was performed. According to these results the largest share was solid DF - tablets and dragee (71 \%) due to their convenience for the population; syrups and oral solutions mainly used in pediatric practice were $10.2 \%$ and $6.3 \%$, respectively; solutions for injection had the smallest part of the domestic pharmaceutical market (4.7\%), but their importance should not be underestimated in cases of immediate type allergic reactions (shock, collapse, Quincke's edema) $[10,11,18]$. The results are presented in Fig. 1.

Table

THE LIST OF AHD PRESENTED AT THE PHARMACEUTICAL MARKET OF UKRAINE

\begin{tabular}{|c|c|c|c|c|c|}
\hline INN & $\begin{array}{l}\text { Name of the } \\
\text { medicinal } \\
\text { product }\end{array}$ & $\begin{array}{l}\text { Pharmaceutical } \\
\text { form }\end{array}$ & Manufacturer & $\begin{array}{l}\text { Retail } \\
\text { price } \\
\text { (UAH) }\end{array}$ & $\begin{array}{l}\text { Ca.s. } \\
(\%)\end{array}$ \\
\hline 1 & 2 & 3 & 4 & 5 & 6 \\
\hline $\begin{array}{l}\text { Dextromethorphan } \\
\text { hydrobromide + } \\
\text { Levocetirizine dihy- } \\
\text { drochloride }\end{array}$ & Tsetlo Plus & Tablets & $\begin{array}{l}\text { Evertogen Life Science Li- } \\
\text { mited, India }\end{array}$ & $\mathrm{N} / \mathrm{A}$ & $\mathrm{N} / \mathrm{A}$ \\
\hline $\begin{array}{l}\text { Montelukast, Levo- } \\
\text { cetirizine dihydro- } \\
\text { chloride }\end{array}$ & Alerhinol Plus & Tablets & $\begin{array}{l}\text { Bafna Pharmaceuticals Ltd., } \\
\text { India }\end{array}$ & 364,00 & 3.39 \\
\hline \multirow[t]{2}{*}{$\begin{array}{l}\text { Diphenhydramine } \\
\text { hydrochloride }\end{array}$} & $\begin{array}{l}\text { Dimedrol-Dar- } \\
\text { nitsa }\end{array}$ & $\begin{array}{l}\text { Tablets, Solution } \\
\text { for injections }\end{array}$ & $\begin{array}{l}\text { JSC “Darnytsia Pharmaceuti- } \\
\text { cal Firm", Ukraine }\end{array}$ & 105,35 & 0.98 \\
\hline & Dimedrol & $\begin{array}{l}\text { Solution for } \\
\text { injections }\end{array}$ & JSC Galichfarm, Ukraine & 70,00 & 0.65 \\
\hline Dimenhydrinate & Dramina & Tablets & $\begin{array}{l}\text { Jadran-Galenski Laboratory } \\
\text { dd. Croatia }\end{array}$ & 217,88 & 2.03 \\
\hline $\begin{array}{l}\text { Clemastine fuma- } \\
\text { rate }\end{array}$ & Tavegil & \begin{tabular}{|l|} 
Solution for in- \\
jections, Tablets
\end{tabular} & $\begin{array}{l}\text { Takeda Austria GmbH, } \\
\text { Austria }\end{array}$ & 176,75 & 1.65 \\
\hline \multirow{4}{*}{$\begin{array}{l}\text { Dimethyndene } \\
\text { maleate }\end{array}$} & Fenokit & Oral drops & JSC Sperko Ukraine, Ukraine & 308,00 & 2.87 \\
\hline & $\begin{array}{l}\text { Dimetinden- } \\
\text { Zdorovie }\end{array}$ & $\begin{array}{l}\text { Oral drops, } \\
\text { Tablets }\end{array}$ & $\begin{array}{l}\text { Pharmaceutical company } \\
\text { "Zdorovie", Ukraine }\end{array}$ & 282,63 & 2.63 \\
\hline & Edermik & Oral drops & JSC "Farmak", Ukraine & 280,00 & 2.61 \\
\hline & Fenistyl & Oral drops & $\begin{array}{l}\text { GSK Consumer Healthcare } \\
\text { SA, Switzerland } \\
\end{array}$ & 630,00 & 5.87 \\
\hline \multirow[t]{3}{*}{$\begin{array}{l}\text { Chloropyramine } \\
\text { hydrochloride }\end{array}$} & Suprastin & $\begin{array}{l}\text { Solution for in- } \\
\text { jections, Tablets }\end{array}$ & $\begin{array}{l}\text { JSC Egis Pharmaceutical } \\
\text { Plant, Hungary }\end{array}$ & 122,50 & 1.14 \\
\hline & $\begin{array}{l}\text { Chloropyramine } \\
\text { Hydrochloride }\end{array}$ & $\begin{array}{l}\text { Solution for } \\
\text { injections }\end{array}$ & $\begin{array}{l}\text { GNCLS Research Plant, } \\
\text { Limited Liability Company, } \\
\text { Ukraine }\end{array}$ & 385,00 & 3.59 \\
\hline & Suprostylin & Tablets & $\begin{array}{l}\text { JSC "Lekhim - Kharkiv", } \\
\text { Ukraine }\end{array}$ & 52,50 & 0.49 \\
\hline \multirow[t]{4}{*}{$\begin{array}{l}\text { Cetirizine dihydro- } \\
\text { chloride }\end{array}$} & Rolinoz & $\begin{array}{l}\text { Tablets, Oral } \\
\text { drops }\end{array}$ & Abc Farmaceutici Spa, Italy & 175,00 & 1.63 \\
\hline & Amertyl & Tablets & Biopharm Co., Ltd., Poland & 123,55 & 1.15 \\
\hline & Zodak & $\begin{array}{l}\text { Oral drops, } \\
\text { Tablets }\end{array}$ & $\begin{array}{l}\text { Ltd. "Zentiva” Czech Repub- } \\
\text { lic }\end{array}$ & 159,95 & 1.49 \\
\hline & Allertec & Tablets & $\begin{array}{l}\text { Pharmaceutical plant “Pol- } \\
\text { pharma" SA, Poland }\end{array}$ & 151,20 & 1.41 \\
\hline
\end{tabular}


Continuation of Table

\begin{tabular}{|c|c|c|c|c|c|}
\hline 1 & 2 & 3 & 4 & 5 & 6 \\
\hline & Cetrin & Tablets & $\begin{array}{l}\text { Dr. Reddy's Laboratories } \\
\text { Ltd., India }\end{array}$ & 126,18 & 1.18 \\
\hline & Cetirizine-Teva & Tablets & Merkle GmbH, Germany & 106,05 & 0.99 \\
\hline & $\begin{array}{l}\text { Cetyrysine- } \\
\text { Astrapharm }\end{array}$ & Tablets & Ltd. "Astrafarm”, Ukraine & 90,30 & 0.84 \\
\hline \multirow[t]{20}{*}{$\begin{array}{l}\text { Levocetirizine dihy- } \\
\text { drochloride }\end{array}$} & Hlentset & Tablets & $\begin{array}{l}\text { Glenmark Pharmaceuticals } \\
\text { Ltd., India }\end{array}$ & 103,25 & 0.96 \\
\hline & Tsezera & Tablets & $\begin{array}{l}\text { KRKA, dd, Novo mesto, } \\
\text { Slovenia }\end{array}$ & 525,00 & 4.89 \\
\hline & Zilola & Tablets & $\begin{array}{l}\text { Ltd. “Gedeon Richter Po- } \\
\text { land", Poland }\end{array}$ & 174,65 & 1.63 \\
\hline & Alerholik & $\begin{array}{l}\text { Tablets, Oral } \\
\text { drops }\end{array}$ & JSC "Technolog", Ukraine & 192,50 & 1.79 \\
\hline & Tsetrylev Neo & Tablets & Hetero Labs Limited, India & 126,00 & 1.17 \\
\hline & Allervey & Tablets & $\begin{array}{l}\text { Dr. Reddy's Laboratories } \\
\text { Ltd., India }\end{array}$ & 178,50 & 1.66 \\
\hline & Levzirine & Tablets & Hetero Labs Limited, India & 110,25 & 1.03 \\
\hline & $\begin{array}{l}\text { Kontrahist } \\
\text { Alerdzhi }\end{array}$ & Tablets & $\begin{array}{l}\text { JSC "Adamed Pharma", } \\
\text { Poland }\end{array}$ & 110,25 & 1.03 \\
\hline & Cetrimac & Tablets & $\begin{array}{l}\text { MacLeods Pharmaceuticals } \\
\text { Limited, India }\end{array}$ & 99,75 & 0.93 \\
\hline & $\begin{array}{l}\text { Levocetyryzine- } \\
\text { Astrapharm }\end{array}$ & Tablets & Ltd. “Astrafarm” Ukraine & 134,75 & 1.26 \\
\hline & Tsetlo & Tablets & $\begin{array}{l}\text { Evertogen Life Science Lim- } \\
\text { ited, India }\end{array}$ & $\mathrm{N} / \mathrm{A}$ & N/A \\
\hline & Allergofree & Tablets & $\begin{array}{l}\text { Simpex Pharma Pvt. Ltd., } \\
\text { India }\end{array}$ & 262,50 & 2.45 \\
\hline & Lasin & Tablets & Hetero Labs Limited, India & $\mathrm{N} / \mathrm{A}$ & $\mathrm{N} / \mathrm{A}$ \\
\hline & L-May & Oral drops & JSC Sperko Ukraine, Ukraine & 194,25 & 1.81 \\
\hline & Erhotsetal & Tablets & $\begin{array}{l}\text { JSC "Kyiv vitamin factory", } \\
\text { Ukraine }\end{array}$ & 103,25 & 0.96 \\
\hline & Aleron & Tablets & $\begin{array}{l}\text { Emcure Pharmaceuticals } \\
\text { Limited, India }\end{array}$ & 110,25 & 1.03 \\
\hline & L-Cet & Tablets, Syrup & $\begin{array}{l}\text { Kusum Healthcare PVT LTD, } \\
\text { India }\end{array}$ & 105,00 & 0.98 \\
\hline & Tsetrylev & Tablets, Syrup & FDS Limited, India & 123,20 & 1.15 \\
\hline & Ksyzal & Tablets & $\begin{array}{l}\text { UCB Farchim SA, Switzer- } \\
\text { land }\end{array}$ & 1750,00 & 16.31 \\
\hline & Alerzine & $\begin{array}{l}\text { Tablets, Oral } \\
\text { drops }\end{array}$ & $\begin{array}{l}\text { JSC Egis Pharmaceutical } \\
\text { Plant, Hungary }\end{array}$ & 183,40 & 1.71 \\
\hline $\begin{array}{l}\text { Cyproheptadine } \\
\text { hydrochloride }\end{array}$ & Perytol & Tablets & $\begin{array}{l}\text { JSC Egis Pharmaceutical } \\
\text { Plant, Hungary }\end{array}$ & 169,40 & 1.58 \\
\hline \multirow[t]{6}{*}{ Loratadine } & $\begin{array}{l}\text { Loratadine- } \\
\text { Zdorovie }\end{array}$ & Tablets, Syrup & $\begin{array}{l}\text { Pharmaceutical company } \\
\text { "Zdorovie", Ukraine }\end{array}$ & 39,38 & 0.37 \\
\hline & Lorizan & Tablets & $\begin{array}{l}\text { JSC “Kievmedpreparat", } \\
\text { Ukraine }\end{array}$ & 89,25 & 0.83 \\
\hline & Claritin & Tablets, Syrup & $\begin{array}{l}\text { Schering Plough Labo NV, } \\
\text { Belgium }\end{array}$ & 71,75 & 0.67 \\
\hline & $\begin{array}{l}\text { Loratadine- } \\
\text { Darnitsa }\end{array}$ & Tablets & $\begin{array}{l}\text { JSC "Pharmaceutical com- } \\
\text { pany "Darnitsa", Ukraine }\end{array}$ & 39,90 & 0.37 \\
\hline & Loratadine & Tablets & Ltd. "Astrafarm" Ukraine & 28,00 & 0.26 \\
\hline & Loratadine & Tablets & $\begin{array}{l}\text { JSC "Lekhim - Kharkiv", } \\
\text { Ukraine }\end{array}$ & 23,45 & 0.22 \\
\hline
\end{tabular}


Continuation of Table

\begin{tabular}{|c|c|c|c|c|c|}
\hline 1 & 2 & 3 & 4 & 5 & 6 \\
\hline & Loratadine & Tablets & JSC "Farmak" Ukraine & 32,90 & 0.31 \\
\hline & Erolin & Tablets & $\begin{array}{l}\text { JSC EGIS Pharmaceutical } \\
\text { Plant, Hungary }\end{array}$ & 218,75 & 2.04 \\
\hline & Lorano & $\begin{array}{l}\text { Oral Suspension, } \\
\text { Tablets, ODT } \\
\text { Tablets } \\
\end{array}$ & $\begin{array}{l}\text { Sandoz Pharmaceuticals DD, } \\
\text { Slovenia }\end{array}$ & 250,25 & 2.33 \\
\hline & Loratadine & Tablets & $\begin{array}{l}\text { JSC "Kievmedpreparat", } \\
\text { Ukraine }\end{array}$ & 45,50 & 0.42 \\
\hline & Aleric & Tablets & Us Pharma Ltd., Poland & 252,00 & 2.35 \\
\hline & Lorfast & Tablets & $\begin{array}{l}\text { Cadila Pharmaceuticals } \\
\text { Limited, India }\end{array}$ & $\mathrm{N} / \mathrm{A}$ & $\mathrm{N} / \mathrm{A}$ \\
\hline & $\begin{array}{l}\text { Loratadine- } \\
\text { Stoma }\end{array}$ & Tablets & JSC "Stoma", Ukraine & 43,75 & 0.41 \\
\hline & Loratadine & Syrup & $\begin{array}{l}\text { DKP Pharmaceutical Factory } \\
\text { LLC, Ukraine }\end{array}$ & 117,60 & 1.10 \\
\hline \multirow[t]{4}{*}{ Mebhydrolin } & Diazoline & Tablets, Dragee & JSC "Farmak" Ukraine & 28,18 & 0.26 \\
\hline & $\begin{array}{l}\text { Diazoline-Dar- } \\
\text { nitsa }\end{array}$ & Tablets & $\begin{array}{l}\text { JSC “Pharmaceutical com- } \\
\text { pany “Darnitsa", Ukraine }\end{array}$ & 29,75 & 0.28 \\
\hline & $\begin{array}{l}\text { Diazolin-SB- } \\
\text { PHARMA }\end{array}$ & Dragee & JSC "Vitamins" Ukraine & 28,35 & 0.26 \\
\hline & $\begin{array}{l}\text { Diazoline For } \\
\text { Children }\end{array}$ & $\begin{array}{l}\text { Granules for oral } \\
\text { suspension }\end{array}$ & $\begin{array}{l}\text { GNCLS Research Plant, } \\
\text { Limited Liability Company, } \\
\text { Ukraine }\end{array}$ & 105,00 & 0.98 \\
\hline \multirow[t]{4}{*}{$\begin{array}{l}\text { Ketotifen hydrofu- } \\
\text { marate }\end{array}$} & Ketotifen & Syrup & $\begin{array}{l}\text { Borschagovsky Chemical } \\
\text { and Pharmaceutical Plant } \\
\text { Scientific and Production } \\
\text { Center, Ukraine }\end{array}$ & 110,43 & 1.03 \\
\hline & Ketotifen & Tablets & $\begin{array}{l}\text { GNCLS Research Plant, } \\
\text { Limited Liability Company, } \\
\text { Ukraine }\end{array}$ & 14,35 & 0.13 \\
\hline & Ketotifen & Tablets & $\begin{array}{l}\text { JSC "Lekhim - Kharkiv", } \\
\text { Ukraine }\end{array}$ & 25,03 & 0.23 \\
\hline & $\begin{array}{l}\text { Ketotifen So- } \\
\text { farma }\end{array}$ & Tablets & JSC “Vitamins”, Ukraine & 26,25 & 0.24 \\
\hline \multirow[t]{5}{*}{$\begin{array}{l}\text { Fexofenadine hy- } \\
\text { drochloride }\end{array}$} & $\begin{array}{l}\text { Feksofen- } \\
\text { Sanovel } \\
\end{array}$ & Tablets & $\begin{array}{l}\text { Sanovel Ilac Sanayi ve } \\
\text { Ticaret AS, Turkey } \\
\end{array}$ & $\mathrm{N} / \mathrm{A}$ & $\mathrm{N} / \mathrm{A}$ \\
\hline & Tigofast & Tablets & $\begin{array}{l}\text { Flamingo Pharmaceuticals } \\
\text { Ltd., India } \\
\text { Arthur Pharmaceuticals Pvt. } \\
\text { Ltd., India }\end{array}$ & 227,50 & 2.12 \\
\hline & Altiva & Tablets & $\begin{array}{l}\text { Sun Pharmaceutical Indus- } \\
\text { tries Limited, India }\end{array}$ & $\mathrm{N} / \mathrm{A}$ & $\mathrm{N} / \mathrm{A}$ \\
\hline & Fexofast & Tablets & Micro Labs Limited, India & 194,25 & 1.81 \\
\hline & Allegra & Tablets & $\begin{array}{l}\text { Sanofi Winthrop Industrie - } \\
\text { Tours, France }\end{array}$ & 352,45 & 3.29 \\
\hline \multirow[t]{5}{*}{ Desloratadine } & Allergostop & Tablets & JSC "Fitofarm", Ukraine & 68,25 & 0.64 \\
\hline & Allergomax & Tablets, Syrup & $\begin{array}{l}\text { Pharmaceutical company } \\
\text { "Zdorovie", Ukraine }\end{array}$ & 105,88 & 0.99 \\
\hline & Lordes & Syrup, Tablets & $\begin{array}{l}\text { Nobel Ilac Sanayi is also } \\
\text { ticaret A.S., Turkey }\end{array}$ & 197,75 & 1.84 \\
\hline & Eridez-Darnitsa & Tablets & $\begin{array}{l}\text { JSC "Pharmaceutical com- } \\
\text { pany “Darnitsa", Ukraine }\end{array}$ & 176,75 & 1.65 \\
\hline & Alersis & $\begin{array}{l}\text { Oral Solution, } \\
\text { Tablets }\end{array}$ & $\begin{array}{l}\text { Laboratorios Normon SA, } \\
\text { Spain }\end{array}$ & $\mathrm{N} / \mathrm{A}$ & $\mathrm{N} / \mathrm{A}$ \\
\hline
\end{tabular}


Continuation of Table

\begin{tabular}{|c|c|c|c|c|c|}
\hline 1 & 2 & 3 & 4 & 5 & 6 \\
\hline & Desloratadine & Tablets, Syrup & JSC "Technolog", Ukraine & 137,90 & 1.29 \\
\hline & Alerdez & Syrup, Tablets & $\begin{array}{l}\text { Borschagovsky Chemical } \\
\text { and Pharmaceutical Plant } \\
\text { Scientific and Production } \\
\text { Center, Ukraine }\end{array}$ & 109,90 & 1.02 \\
\hline & Eol & Oral solution & $\begin{array}{l}\text { Stada Arzneimittel AG, } \\
\text { Germany }\end{array}$ & N/A & N/A \\
\hline & Blogir-3 & $\begin{array}{l}\text { Tablets, Oral } \\
\text { solution }\end{array}$ & $\begin{array}{l}\text { Belupo, medicine and cos- } \\
\text { metics, dd, Croatia }\end{array}$ & $\mathrm{N} / \mathrm{A}$ & N/A \\
\hline & Desradine & Tablets & $\begin{array}{l}\text { KRKA, dd, Novo mesto, } \\
\text { Slovenia }\end{array}$ & 227,50 & 2.12 \\
\hline & Hitaksa & $\begin{array}{l}\text { Oral solution, } \\
\text { Tablets, ODT } \\
\text { Tablets }\end{array}$ & $\begin{array}{l}\text { Famar A.V.E. Avalon Plant, } \\
\text { Greece }\end{array}$ & 192,50 & 1.79 \\
\hline & $\begin{array}{l}\text { Desloratadine- } \\
\text { Astrapharm }\end{array}$ & Tablets & Ltd. "Astrafarm” Ukraine & 107,10 & 1.00 \\
\hline & Eslotin & Tablets & Word Medical A.S., Turkey & 157,50 & 1.47 \\
\hline & Alleric Neo & Oral solution & $\begin{array}{l}\text { Famar Ave Avalon Factory } \\
49 \mathrm{~km} \text { Athens-Lamia State } \\
\text { Road, Greece } \\
\end{array}$ & N/A & $\mathrm{N} / \mathrm{A}$ \\
\hline & Free-Al & Tablets & $\begin{array}{l}\text { Saneca Pharmaceuticals JSC, } \\
\text { Slovak Republic }\end{array}$ & 126,35 & 1.18 \\
\hline & Allergosan & $\begin{array}{l}\text { Tablets, Oral } \\
\text { solution }\end{array}$ & JSC "Sofarma", Bulgaria & $\mathrm{N} / \mathrm{A}$ & N/A \\
\hline & Erius & Tablets, Syrup & $\begin{array}{l}\text { Schering Plough Labo NV, } \\
\text { Belgium }\end{array}$ & 980,00 & 9.14 \\
\hline & Freebris & Syrup & $\begin{array}{l}\text { Gracure Pharmaceuticals } \\
\text { LTD, India }\end{array}$ & $\mathrm{N} / \mathrm{A}$ & $\mathrm{N} / \mathrm{A}$ \\
\hline & Edem & Syrup, Tablets & JSC "Farmak" Ukraine & 159,25 & 1.48 \\
\hline Bilastine & Nixar & Tablets & Faes Farma, SA, Spain & 277,90 & 2.59 \\
\hline $\begin{array}{l}\text { Hyfenadine hydro- } \\
\text { chloride }\end{array}$ & Fencarol & Tablets & JSC "Olaynfarm" Latvia & 246,75 & 2.30 \\
\hline $\begin{array}{l}\text { Sehifenadine hy- } \\
\text { drochloride }\end{array}$ & Gistaphen & Tablets & JSC “Olaynfarm” Latvia & 422,45 & 3.94 \\
\hline \multicolumn{4}{|l|}{ Average } & 189,37 & 1.77 \\
\hline
\end{tabular}

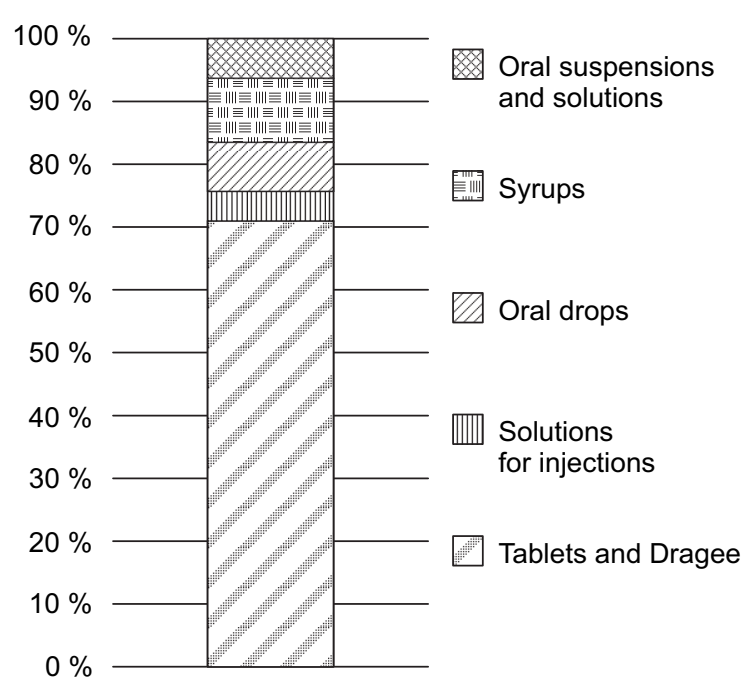

Fig. 1. Distribution of the AHD market by dosage forms
The analysis of AHD by the manufacturing countries revealed that almost $2 / 3$ of the AHD market is occupied by imported drugs and only $1 / 3$ by the Ukrainian ones (Fig. 2). Moreover, in 2014 , this indicator was $50 \%$ / $50 \%$, indicating

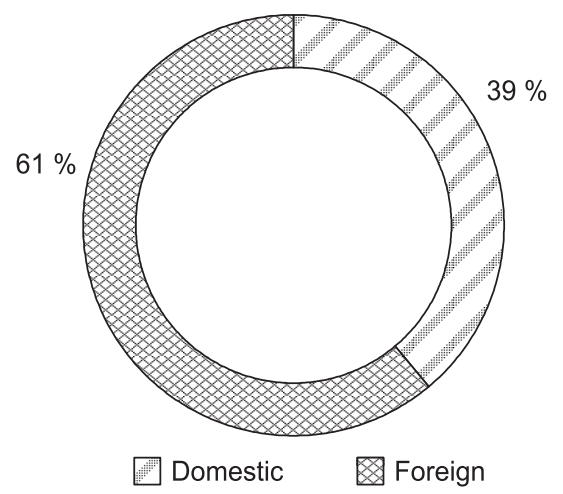

Fig. 2. The share of foreign and domestic AHD 


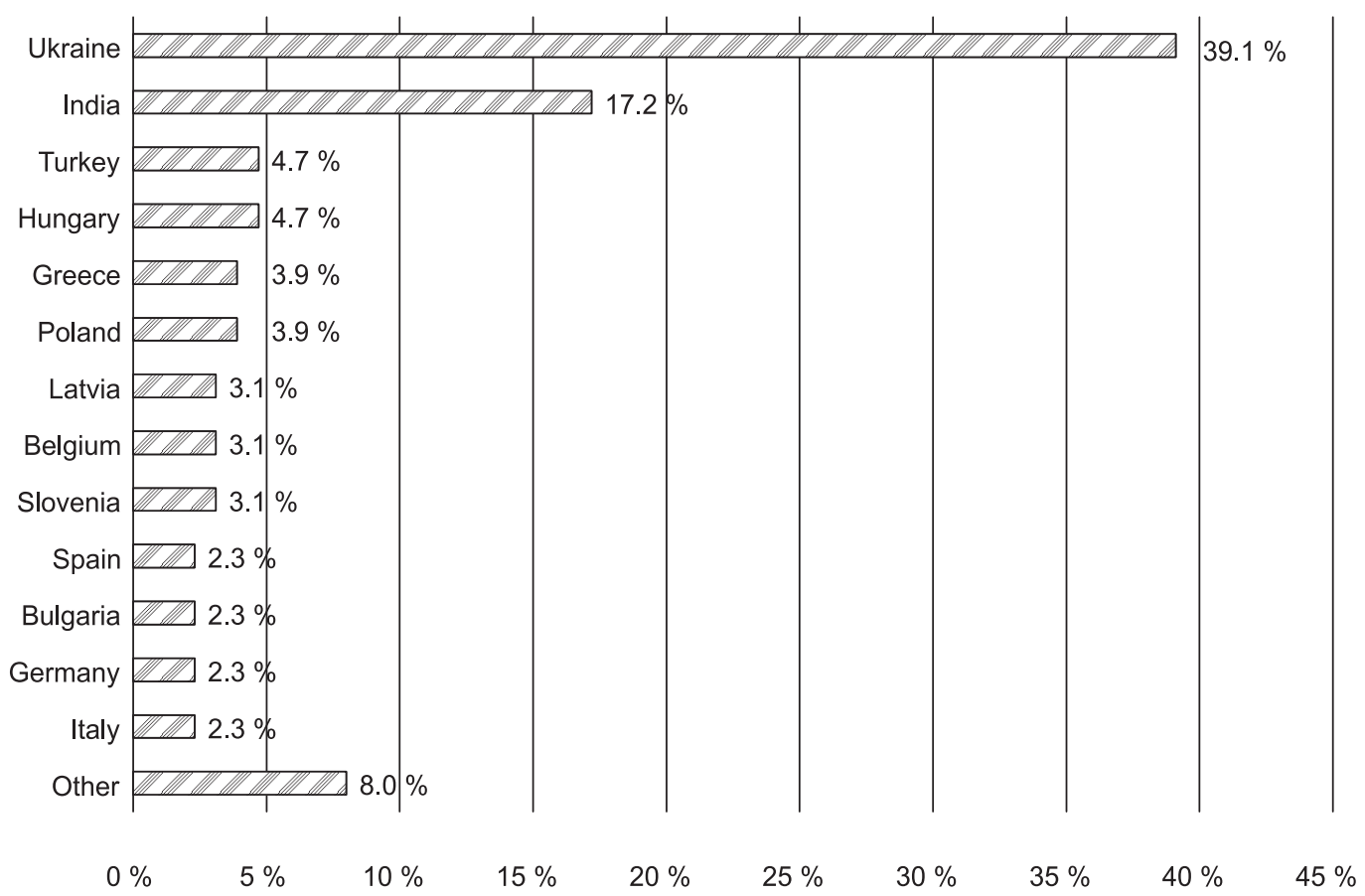

Fig. 3. Distribution of the AHD market by manufacturing countries

a decrease of the share of the Ukrainian manufacturers in the segment of the pharmaceutical market studied $[12,18]$.

Among the foreign manufacturers, India had a stable leading position (17.2\%). The total contribution of the European Union countries was $39 \%$, among them the largest share $(16.4 \%)$ was in the countries of Eastern and Southern Europe (Hungary, Greece, Poland, Latvia) (Fig. 3) [18].

According to the results of marketing analysis of this group of drugs for 2014 the following distribution of AHD by international non-proprietary names (INNs) was observed at the pharmaceutical market of Ukraine. Loratadine $(21 \%)$ held the leading position among the trade names (TN) registered. The 2 nd, 3rd and 4 th positions were taken by Desloratadine (16\%), Levocetirizine (15\%) and Cetirizine (13\%) (Fig. 4) [12, 13].

In 5 years (as of 2019) the following changes were observed: Loratadine lost $6 \%$ of the total amount of the TN registered and its leading position; the first positions were taken by Desloratadine, which number of TN increased by $9 \%$ and was $25 \%$, and Levocetirizine with the of number TN increased by $5 \%$ and reached the mark of $20 \%$ (Fig. 5) [18].

The data obtained show an increase in quantity of the third-generation drugs (Levocetirizine, Desloratadine), indicating that health professionals and patients are increasingly guided by the safety profile considerations when choosing a course of treatment of allergic diseases.
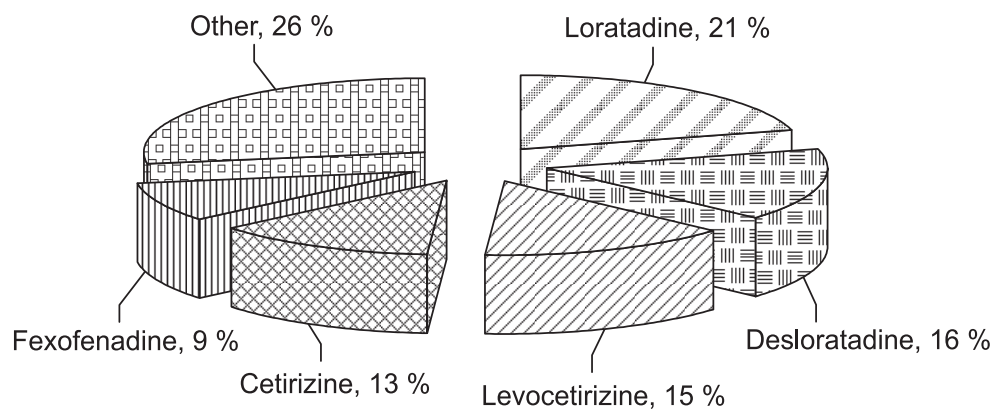

Fig. 4. Distribution of the AHD market by INN as of 2014 


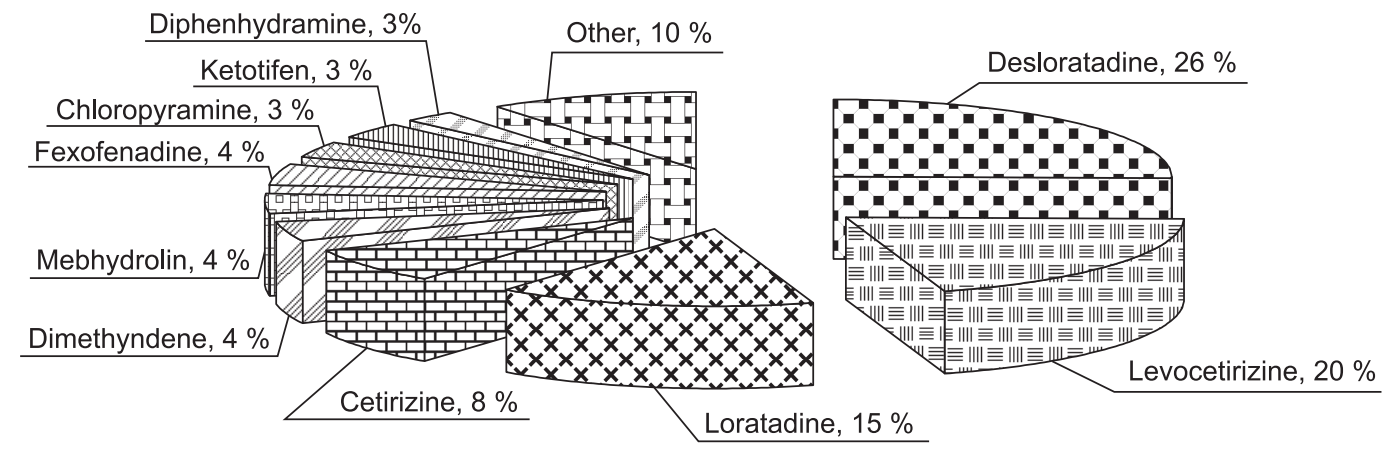

Fig. 5. Distribution of the AHD market by INN as of November 2019

The long-term administration and frequent need for treating children also contribute to the choice of safer drugs [6].

It is important to note that there are no plant-based anti-allergic drugs at the Ukrainian pharmaceutical market.

In accordance with the WHO statistics about $80 \%$ of the world's population prefers drugs based on the plant components [20]. Herbal medicines have several advantages over synthetic ones, first of all, due to their complex action on several links of the pathogenesis of the disease. If the potent substances, such as alkaloids or cardiac glycosides, are absent in the plant raw material, the action of the herbal products is soft and safe, allowing them to be used for a long time [20,21].

The economic factor is significant for the population of our country. Socio-political changes in recent years have led to a decrease in the standard of living of the population and have made medicines (especially imported) unaffordable to the majority of the population.

To account for this factor, the analysis of the AHD market was performed using the solvency index reflecting a percentage of the average monthly disposable income required for the AHD treatment course (1).

In order to obtain the unified results the study of affordability was performed among solid dosage forms - tablets and dragee presented the most widely at the domestic market of AHD.

The results of the calculations are presented in Table.

According to the results in Table the most of AHD are highly affordable for the working population of Ukraine. The Swiss drug "Fenistil" and the Belgian drug "Erius" have the medium affordability. The drug "Xysal" of the Swiss production has appeared to be unaffordable, its average treatment course costs about 1750,00 UAH. The average value of Ca.s is $1.77 \%$; it indicates the availability of the AHD market in total.

\section{Conclusions and prospects for further} research

1. The analysis of the literature sources has revealed the high incidence of allergic diseases among the population of Ukraine.

2. By analyzing the assortment of antihistamine drugs it has been found that the most common dosage form of AHD is a solid form tablets and dragee; their number is $71 \%$ of the total drugs registered.

3 . Based on the results of the marketing research there is a tendency of increasing a number of the third-generation AHD and decreasing a number of older generations; it indicates the importance of the safety profile in the treatment of various types of allergies.

4. According to the results of the AHD affordability study based on the methodology recommended by the WHO a high availability of the most AHD for the working population of Ukraine has been determined.

5 . Over the last 5 years, there has been a decrease in the share of the domestic manufacturers (by $11 \%$ ) at the pharmaceutical market of Ukraine; it indicates the import dependence of the market and the need to create new domestic AHD.

6 . The absence of the plant-based anti-allergic drugs at the pharmaceutical market of Ukraine has been found; it indicates the prospect for further research in the direction of developing an herbal medicine for the systemic treatment of allergic diseases.

Conflict of interests: authors have no conflict of interests to declare. 


\section{References}

1. Белая книга по аллергии Всемирной организации по аллергии / под ред. R. Pawankar, G. W. Canonica, S. T. Holgate, R. F. Lockey. World Allergy Organization. 2012. № 3 (52). C. 33-41.

2. Особенности распространения аутоиммунной крапивницы в западной Грузии / И. Пхакадзе и др. Аллергология и иммунология. 2019. Т. 20, № 1. С. 14-16.

3. Гацкая Д. О., Корицкая И. В. Динамика распространенности аллергических заболеваний среди детей и молодежи Винницкой области (результаты 1-го этапа эпидемиологического исследования). Астма та Алергія. 2015. № 1. С. 17-22.

4. Пухлик Б. М. Аллергия - проблема не только аллергологов. Запорожский медицинский журнал. 2011. Т. 13, № 2. С. 108-110.

5. Schuler Iv. C. F., Montejo M. J. Allergic Rhinitis in Children and Adolescents. Pediatr Clin N Am. 2019. 66. P. 981-993. doi: 10.1016/j.pcl.2019.06.004.

6. Apel K. Home environment: respiratory and allergic phenotypes from birth to age six in the PELAGIE cohort.npj Primary Care Respiratory Medicine. 2019. Vol. 29:29.P. 1-8. doi: 10.1038/s41533-019-0141-y.

7. Global Atlas of Allergy / ed. Cezmi A. Akdis, Ioana Agache. EAACI. 2014. 388 p.

8. Карева Е. Н. Выбор антигистаминного препарата: взгляд фармаколога. РМЖ. Медищинское обозрение. 2016. №12. С. 811-816.

9. Лусс Л. В. Выбор антигистаминных препаратов в лечении аллергических и псевдоаллергических реакций. ГНЦ «Институт иммунологии ФМБА РФ», г. Москва. Астма та алергія. 2010. № 1-2. С. 70-76.

10. Клінічна фармація : підруч. для студ. вищ. навч. закл. / за ред. В. П. Черних, І. А. Зупанця, I. Г. Купновицької. Харків : НФаУ : Золоті сторінки, 2013. 912 с.

11. Лусс Л. В., Ильина Н. И. Антигистаминные препараты в общеклинической практике: Вопросы и ответы. В помощь практическому врачу. 2017. 184 с.

12. Iakovlieva L. V., Berdnik O. G., Gurtyakova A. O. Analysis of assortment and volumes of consumption of antihistamine preparations in Ukraine. Pharmaceutical journal, 2018, № 1-2. P. 12-21.

13. Яковлєва Л. В., Сизенко М. О. Аналіз антигістамінних препаратів на українському фармацевтичному ринку. Фармацевтичний журнал, 2014. С. 277.

14. Яковлєва Л. В., Передерій А. В. Доступність антигістамінних засобів для українських пацієнтів у 2013-2014 роках. Управління, економіка та забезпечення якості в фармації. 2015. № 4 (42). C. 87-94.

15. Лукьянчук Е. Частые ОРВИ и аллергия: есть ли связь? Еженедельник аптека. 2013. № 4 (875). URL: http://www.apteka.ua/article/202653 (дата звернення: 03.03.2020).

16. Державна служба статистики України. URL: http://ukrstat.gov.ua/ (дата звернення: 03.03.2020).

17. Яковлева Л. В., Кириченко Н. О., Лукашевич П. Ю. Оценка доступности препаратов метформина и гликлазида на фармацевтическом рынке Украины. Ендокринологія. 2016. Т. 21, № 2. C. 119-124. URL: http://nbuv.gov.ua/UJRN/enkrl_2016_21_2_6 (дата звернення: 03.03.2020).

18. Державний реєстр лікарських засобів України. URL:http://www.drlz.com.ua/ (дата звернення: 03.03.2020).

19. Справочник «Компендіум. Лікарські препарати». URL: https://compendium.com.ua/ (дата звернення: 03.03.2020).

20. Шостак Т. А., Калинюк Т. Г., Гудзь Н. І. Особливості фармацевтичної розробки рослинних препаратів (Огляд літератури). Фітотерапія. Часопис. 2014. № 4. С. 77-82.

21. Шостак Л. Г., Пальчик К. О. Мікро- та макроелементний склад основних органів фікусу бенджаміна. Молодий вчений. 2018. № 3 (55). С. 425-429.

22. The role of Histamine and Histamine Receptors in Mast Cell-Mediated Allergy and Inflammation / E. B. Thangam et al. The hunt for New Therapeutic Targets. Front. Immunol. 2018. Vol. 9:1873.P. 1-9. doi: 10.3389/fimmu.2018.01873.

23. Comprehensive medical videolibrary. URL: http://www.Osmosis.org (дата звернення: 03.03.2020).

24. Клиническая фармакология по Гудману и Гилману : в 4 т. / под общ. ред. Г. Гилмана ; ред. Дж. Хардман, Л. Лимберд ; пер. с англ. Москва : Практика, 2006. 336 с.

25. Зайков С. В. Эффективность антигистаминных препаратов при ОРВИ у больных аллергическими заболеваниями. Алергологія. Погляд фахівця. 2013. С. 43. 


\section{References}

1. Pawankar, R., Canonica, G. W., Holgate, S. T., Lockey, R. F. (Eds.). (2012). White book of World Allergy Organization. World Allergy Organization, 3 (52), 33-41.

2. Phakadze, I, Shavlakadze, N., Lezhava, D., Silagadze, A., Chkhikvadze, L. (2019). Allergology and Immunology, 20 (1), 14-16.

3. Hatskaia, D. O., Koritskaia, I. V. (2015). Asthma and Allergy, 1, 17-22.

4. Pukhlik, B. M. (2011). Zaporozhskii meditsinskii zhurnal, 13 (2), 108-110.

5. Schuler, Iv. C. F., Montejo, M. J. (2019). Pediatr Clin N Am, 66, 981-993.

6. Apel, K. (2019) npj Primary Care Respiratory Medicine, 29:29, 1-8. doi: 10.1038/s41533-019-0141-y.

7. Cezmi, A. Akdis, Ioana, Agache. (2014). EAACI, 388.

8. Kareva, E. N. (2016). RMJ. Medical Review, 12, 811-816.

9. Luss, L. V. (2010). Asthma and Allergy, 1-2, 70-76.

10. Chernykh, V. P., Zupanets, I. A., Kupnovytska, I. G. (Eds.). (2013). Klinichna farmatsiia. Kharkiv: NFaU: Zoloti Storinki, 912.

11. Luss, L. V., Il'ina, N.I. (2017). V pomoshch prakticheskomu vrachu, 184.

12. Iakovlieva, L. V., Berdnik, O. G., Gurtiakova, A. O. (2018). Pharmaceutical journal, 1-2, 12-21.

13. Iakovlieva, L. V., Sizenko, M. O. (2014). Pharmaceutical Journal, 277.

14. Iakovlieva, L. V., Perederii, A. V. (2016). Management, economy and quality assurance in pharmacy, 87-94.

15. Lukianchuk, E. (2013). Weekly Pharmacy, 4 (875). Available at: http://www.apteka.ua/article/202653 (Date of access: 03.03.20200).

16. State Statistics Service of Ukraine. Available at:http://ukrstat.gov.ua/ (Date of access: 03.03.20200).

17. Iakovlieva, L. V., Kyrichenko, N. O., Lukashevych, P. Y. (2016). Endocrinology, 21 (2), 119-124. Available at: URL: http://nbuv.gov.ua/UJRN/enkrl_2016_21_2_6(Date of access: 03.03.20200).

18. State register of drugs of Ukraine. Available at: http://www.drlz.com.ua/(Date of access: 03.03.20200).

19. Compendium on-line. Available at: https://compendium.com.ua (Date of access: 03.03.20200).

20. Shostak, T. A., Kaliniuk, T. G., Gudz, N. I. (2014). Phytotherapy. The Chronicle,4, 77-82.

21. Shostak, L. G., Palchik, K. O. (2018). Young scientist, 3 (55), 425-429.

22. Thangam, E. B., Jemima, E. A., Singh, H., Baig, M. S., Khan, M., Clinton, B. Mathias, Martin, K. Church, Saluja, R. (2018). The hunt for New Therapeutic Targets. Front. Immunol., 9: 1873, 1-9.

23. Comprehensive medical video library. Available at: http://www.Osmosis.org (Date of access: 03.03.20200).

24. Gilmana, G., Khardman, Dzh., Limberd, L. (Eds.). (2006). Klinicheskaia farmakologiia po Gudmanu i Gilmanu.Moscow: Praktika, 336.

25. Zaikov, S.V. (2013). Allergology. Poglyad fakhivtsya, 43. 
Information about authors:

Andryushayev O. V., postgraduate student of the Department of Industrial Technology of Drugs, National University of Pharmacy (https://orcid.org/0000-0003-3361-7851). E-mail: Linuks454@gmail.com

Ruban O. A., Doctor of Pharmacy (Dr. habil.), professor, head of the Department of Industrial Technology of Drugs,

National University of Pharmacy (http://orcid.org/0000-0002-2456-8210) E-mail: ruban_elen@ukr.net

Masliy Yu. S., Candidate of Pharmacy (Ph. D), associate professor of the Department of Industrial Technology of Drugs,

National University of Pharmacy (http://orcid.org/0000-0002-8968-0262) E-mail: julia.masliy@gmail.com

Iakovlieva L. V., Doctor of Pharmacy (Dr. habil.), professor, head of the Department of Pharmacoeconomics, National University of Pharmacy (http://orcid.org/0000-0002-9961-4664) E-mail: iakovlievalv@gmail.com

Відомості про авторів:

Андрюшаєв О. B., аспірант кафедри заводської технології ліків, Національний фармацевтичний університет (https://orcid.org/0000-0003-3361-7851). E-mail: Linuks454@gmail.com

Рубан О. А., докторка фармацевтичних наук, професорка, завідувачка кафедри заводської технології ліків,

Національний фармацевтичний університет (http://orcid.org/0000-0002-2456-8210). E-mail: ruban_elen@ukr.net

Маслій Ю. С., кандидатка фармацевтичних наук, доцентка кафедри заводської технології ліків, Національний фармацевтичний університет (http://orcid.org/0000-0002-8968-0262). E-mail: julia.masliy@gmail.com

Яковлєва Л. В., докторка фармацевтичних наук, професорка, завідувачка кафедри фармакоекономіки, Національний фармацевтичний університет (http://orcid.org/0000-0002-9961-4664). E-mail: iakovlievalv@gmail.com Сведения об авторах:

Aндрюшаев A. B., аспирант кафедры заводской технологии лекарств, Национальный фармацевтический университет (https://orcid.org/0000-0003-3361-7851).E-mail: Linuks454@gmail.com

Pубан $\boldsymbol{E}$. A., доктор фармацевтических наук, профессор, заведующая кафедрой заводской технологии лекарств, Национальный фармацевтический университет (http://orcid.org/0000-0002-2456-8210). E-mail: ruban_elen@ukr.net Маслий Ю. С., кандидат фармацевтических наук, доцент кафедры заводской технологии лекарств, Национальный фармацевтический университет (http://orcid.org/0000-0002-8968-0262). E-mail: julia.masliy@gmail.com Яковлева Л. В., доктор фармацевтических наук, профессор, заведующая кафедрой фармакоэкономики, Национальный фармацевтический университет (http://orcid.org/0000-0002-9961-4664). E-mail: iakovlievalv@gmail.com 